\title{
Mycobacterial arthritis as a complication of a Bacille Calmette-Guérin (BCG) vaccination in an immunocompetent child B Dérfalvi*1, T Verebély ${ }^{1}$, L Kovács ${ }^{1}$, J Feinberg ${ }^{2}$ and Gy Póder ${ }^{1}$
}

\author{
Address: ${ }^{1} 1$ st Dept. of Pediatrics Semmelweis University, Budapest, Hungary and ${ }^{2}$ Human Genetics of Infectious Diseases, INSERM U550, \\ Université Paris Descartes, Necker Medical School, Paris, France \\ * Corresponding author
}

from I5th Paediatric Rheumatology European Society (PreS) Congress

London, UK. 14-17 September 2008

Published: 15 September 2008

Pediatric Rheumatology 2008, 6(SuppI I):PI36 doi:I0.II86/I546-0096-6-SI-PI36

This abstract is available from: http://www.ped-rheum.com/content/6/SI/PI36

(C) 2008 Dérfalvi et al; licensee BioMed Central Ltd.

BCG vaccine (attenuated strain of virulent Mycobacterium bovis) is worldwide administered in the first weeks of life. Systemic BCG infection or osteomyelitis are rare infrequent complications especially in immunocompromised individuals. We report a case of an immunocompetent infant with BCG arthritis.

A 17 month old boy presented with monoarthritis involving his knee, which started 7 month earlier, responding to NSAID therapy, respectively. On physical examination the only finding was the painful effusion and functional limitation of the right knee, with hyperaemic skin reaction above it. His BCG scar was normal. Laboratory evaluation showed mildly elevated CRP (21 mg/l) and C3. RF, ANA, ENA and serological tests for infections were unrevealing. X-ray showed bony owergrowth of the distal femoral epyphisis without osteomyelitis. Ultrasound and MRI of the knee showed intra- and extraarticular effusion. Aspirates were turbid with $95 \%$ of polymorphs and were sterile with culture, no presence of acid-fast bacilli were detected. Further culture identified BCG vaccine strain of $M$. bovis. Twelve weeks of isoniacid therapy was ineffective, surgical bursectomy gave a complete healing. Histological examination revealed granulomatous inflammation with ceasating necrosis. Hypererg PPD test, normal T-cell functions, NBT-test and analysis of the IFN $\gamma /$ IL-12 pathway ruled out primary immunodeficiency, HIV-test was negative as well.

BCG arthritis (chronic, painful, affecting the bursa) is a rare complication of the vaccine after a long latent period.
Detecting BCG mycobacterium with PCR-reaction from the aspirat could shorten the diagnostic time. Primary immunodeficiencies associated with an increased susceptibility to mycobacterial infections should be ruled out. 